\title{
Simultaneous HPLC Determination of Costunolide and Dehydroco- stuslactone in Xin-ke-shu Preparations
}

\author{
Shu Dong ${ }^{1,2}$, Yue-tao Liu ${ }^{2}$, Jing-bo Peng ${ }^{2}$, Hong-mei Jia ${ }^{2}$, Zhong-mei Zou ${ }^{2 *}$ and Chang-yuan Yu ${ }^{1 *}$ \\ ${ }^{I}$ College of Life Science and Technology, Beijing University of Chemical Technology, Beijing 100029, China \\ ${ }^{2}$ Institute of Medicinal Plant Development, Chinese Academy of Medical Sciences \& Peking Union Medical College, \\ Beijing 100193, China
}

\begin{abstract}
The objective of this study is to establish a quantitative method for simultaneous determination of costunolide and dehydrocostuslactone in Xin-ke-shu preparations. The HPLC quantitative analysis was established on a Hypersil BDS column $(100 \times 4.6 \mathrm{~mm}, 2.4 \mu \mathrm{m}$, Thermo) with UV detection at $225 \mathrm{~nm}$. The mobile phase was water and methanol (30:70, $\mathrm{v} / \mathrm{v})$, and used at a flow rate at $0.5 \mathrm{~mL} \cdot \mathrm{min}^{-1}$. The established method showed a good linearity $\left(R^{2}>0.9999\right)$ over the investigated concentration ranges $(0.07-2.80 \mu \mathrm{g})$, good inter-day and intra-day precisions (less than $3 \%$ ) and good recoveries (from $99.82 \%$ to $99.98 \%$ ) for both target compounds. The method was found to be suitable for simultaneous determination of costunolide and dehydrocostuslactone in Xin-ke-shu preparations and can be used as a comprehensive approach to the previous reported quality control of Xin-ke-shu preparations.
\end{abstract}

Keywords: Xin-ke-shu preparations, costunolide, dehydrocostuslactone, HPLC, quantitation.

\section{INTRODUCTION}

Xin-ke-shu, a traditional Chinese medicine (TCM) preparation composed of Crataegus pinnatifide (Shan-Zha), Radix Salviae Miltiorrhiae (Dan-Shen), Radix Puerariae (Ge-Gen), Radix Notoginseng (San-Qi) and Radix Aucklandiae (Mu-Xiang), has demonstrated significant effects against coronary heart disease with few side-effects [1]. The main treatment for chest tightness, angina, high blood pressure, dizziness, headache, neck pain and arrhythmia, high blood lipids caused by the Qi stagnation and coronary artery disease is applied in clinical trials.

There are reports of puerarin [2], tanshinone IIA [3] and protocatechuic aldehyde [4] as an index of the quality control methods for Xin-ke-shu preparations. In order to quickly screen the chemical profiles and control the quality of Xinke-shu preparations, liquid chromatography coupled with electrospray ionization hybrid linear trap quadrupole orbitrap (LC-LTQ-Orbitrap) mass spectrometry was applied for simultaneous identification and quantitative determination of the constituents in complex chemical system [5]. A total of 51 compounds, including phenolic acids, isoflavone-Cglycosides, isoflavone-O-glycosides, were identified or tentatively deduced. However, none of constituents from the roots of Radix Aucklandiae (Mu-Xiang), one of the main herbs in Xin-ke-shu, were detected. Costunolide and dehydrocostuslactone (structures shown in Fig. 1), the main constituents of the roots of Radix Aucklandiae [6], have been

*Address correspondence to these authors at the Institute of Medicinal Plant Development, Chinese Academy of Medical Sciences \& Peking Union Medical College, Beijing 100193, China; Tel.: 86-10-64448589;

E-mail: yucy@mail.buct.edu.cn; College of Life Science and Technology, Beijing University of Chemical Technology, Beijing 100029, China;

Tel: 86-10-62899756; E-mail: zmzou@implad.ac.cn

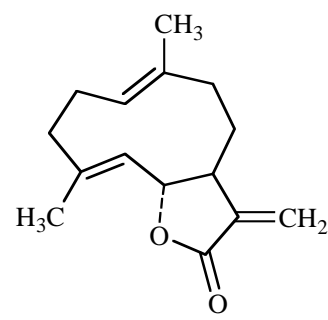

1<smiles>C=C1C(=O)OC2C1CCC(=C)C1CCC(=C)C12</smiles>

2
Fig. (1). The structures of costunolide (1) and dehydrocostuslactone (2).

usually used as chemical markers for quality control of this usually used as chemical markers for quality control of this herb [7]. But both of them could not be detected by using our previously reported LC-MS conditions [5]. In order to provide a complementary strategy for quality control of Xinke-shu preparations, this paper described a validated and optimized HPLC method for simultaneous quantization of costunolide and dehydrocostuslactone in Xin-ke-shu preparations.

\section{MATERIALS AND METHODS}

\section{Chemicals}

HPLC grade methanol was purchased from Fisher (New Jersey, USA) and other reagents and solvents were of analytic grade. HPLC water was prepared with the Millipore Milli-Q SP water purification (Bedford, MA, USA) system.

Costunolide (1) and dehydrocostuslactone (2) were provided by the National Institutes for Food and Drug Control and their purities (>99\%) were determined by HPLC. 
Table 1. A Summary of the Tested Samples

\begin{tabular}{|l|l|l|l|}
\hline \multicolumn{1}{|c|}{ Sample No. } & Preparation Form & \multicolumn{1}{c|}{ Source } & Batch No. \\
\hline \hline Sh1 & Tablet & Shandong Wohua Pharmaceutical Technology Co., Ltd, China & 090629 \\
\hline Sh2 & Tablet & Shandong Wohua Pharmaceutical Technology Co., Ltd, China & 100537 \\
\hline Sh3 & Tablet & Shandong Wohua Pharmaceutical Technology Co., Ltd, China & 100645 \\
\hline Sh4 & Tablet & Shandong Wohua Pharmaceutical Technology Co., Ltd, China & 100104 \\
\hline Sh5 & Tablet & Shandong Wohua Pharmaceutical Technology Co., Ltd, China & 100307 \\
\hline S & Capsule & Sichuan Longren Pharmaceutical Co., Ltd, China & 100301 \\
\hline Ch1 & Capsule & Chongqing Xieran Pharmaceutical Co., Ltd, China & 100301 \\
\hline CH2 & Capsule & Chongqing Xieran Pharmaceutical Co., Ltd, China & 090901 \\
\hline H1 & Capsule & Hebei Guojin Pharmaceutical Co., Ltd, China & 1001051 \\
\hline H2 & Capsule & Hebei Guojin Pharmaceutical Co., Ltd, China & 0904051 \\
\hline X & Capsule & Xian Tianyi Pharmaceutical Co., Ltd, China & $100404-029$ \\
\hline Y & Capsule & Deyuantang Pharmacy Group, China & $90003004-076$ \\
\hline J & Pill & Jilin Jichun Pharmaceutical Co., Ltd, China & 100101 \\
\hline
\end{tabular}

\section{Xin-ke-shu Samples}

A total of 13 samples of Xin-ke-shu preparations produced by different pharmaceutical companies in China were collected from pharmacy (Table 1). The blank sample was the extract of Xin-ke-shu prescription without MuXiang provided by Shandong Wohua Pharmaceutical Technology Co., Ltd, China.

\section{Chromatographic Conditions}

All sample analyses were carried out on a HPLC system consisted of a Waters 600 HPLC with a 486 UV detector and 717 Autosampler. Data were acquired and processed with the Empower () software system. Chromatographic separation was performed on a Thermo BDS HYPERSIL $\mathrm{C}_{18}$ column $(4.6 \mathrm{~mm} \times 100 \mathrm{~mm}, 2.4 \mu \mathrm{m})$. The mobile phase was delivered at a flow rate of $0.5 \mathrm{~mL} / \mathrm{min}$ consisting methanolwater solution $(70: 30, \mathrm{v} / \mathrm{v})$. The column temperature was maintained at $25^{\circ} \mathrm{C}$ and the effluent was monitored at 225 $\mathrm{nm}$.

\section{Preparation of Standard Solutions}

A stock solution containing costunolide and dehydrocostuslactone was prepared in methanol at the same concentration of $2.8 \mathrm{mg} / \mathrm{mL}$ for each of them and stored at $20^{\circ} \mathrm{C}$ prior to use. Stock solutions were diluted with methanol to give a series of working solutions. The range of the concentration was $7-280 \mu \mathrm{g} / \mathrm{mL}$.

\section{Preparation of Sample Solutions}

The dried powders of Xin-ke-shu preparation $(1.0 \mathrm{~g})$ were accurately weighed and transferred into a $50 \mathrm{~mL}$ Erlenmeyer flask. They were extracted with $25 \mathrm{~mL}$ chloroform in an ultrasonic bath for $20 \mathrm{~min}$. After recruitment the weight, aliquots of $5 \mathrm{~mL}$ of supernatant was precisely taken out, and evaporated to dryness under reduced pressure to give a residue. The residue was dissolved in methanol, quantitatively transferred into a volumetric flask and adjusted to a final volume $(5 \mathrm{~mL})$ with methanol. The obtained solution was filtered through $0.45 \mu \mathrm{m}$ syringe filter before HPLC analysis and $10 \mu \mathrm{L}$ was injected. Each sample solution was injected in triplicate.

The blank sample, having the same amount of raw herb equivalent to $1 \mathrm{~g}$ of Xin-ke-shu preparation except $\mathrm{Mu}$ Xiang was prepared and analyzed identically to Xin-ke-shu samples.

\section{Method Validation}

\section{Specificity}

The specificity was determined by injecting the reference standards, the blank and Xin-ke-shu samples to evaluate possible interferences in samples. The sample preparation and chromatographic condition were optimized to guarantee that no interferences incurred at the retention time of the tested compounds.

\section{Calibration Curves}

The calibration curves were prepared at seven different concentration levels. The range of the concentration was 7$280 \mu \mathrm{g} / \mathrm{mL}$. The limit of detection (LOD) and limit of quantification (LOQ) were determined by injecting dilute solutions of the standards with known concentrations. The LOD and LOQ were defined as the signal-to-noise ratio equal to 3 and 10 , respectively.

\section{Precision, Reproducibility, and Stability}

Precision of the method was determined by preparing and assaying 6 replicate samples of Xin-ke-shu (batch No. 100537) on the same day; the same procedure was repeated 
on three consecutive days. The intra-day and inter-day precisions were determined by comparing variations within the same days and the 3 days, respectively.

\section{Recovery}

The spiked samples were analyzed and the recoveries were calculated by comparing the peak area of measured concentration to the peak area of the spiked concentration.

\section{RESULTS AND DISCUSSION}

\section{Selection of Detection Manner and Optimization of Chromatographic Conditions}

First, the MS condition for detection of costunolide and dehydrocostuslactone was optimized. However, the optimized conditions do not suitable for quantization of other constituents in Xin-ke-shu preparations. In addition, both costunolide and dehydrocostuslactone have their maximum absorbance at $225 \mathrm{~nm}$ [7]. Thus, in order to provide a complementary strategy for quality control of Xin-ke-shu preparations, a simple HPLC method with UV detection was established for simultaneous determination of costunolide and dehydrocostuslactone in Xin-ke-shu preparations.

Various compositions of mobile phase such as acetonitrile-water, methanol-water and acetonitrile-aqueous formic acid, were tried to obtain chromatograms with good resolution of adjacent peaks. The mixture of methanol and water $(70: 30, \mathrm{v} / \mathrm{v})$ were found to give the desired separation in a relatively short analytical time for the HPLC quantization of the two markers.

\section{Optimization of Sample Preparation}

Optimization of the extraction solvents and procedures was essential in the development of analytical methods for herbal extracts because different solvents and procedures will influence the extraction efficiency of different compounds, as well as the overall sensitivity and selectivity of the method.

Variables involved in the extraction such as solvents (ethyl acetate, methanol and chloroform), extraction methods (sonication and refluxing) and extraction time (10, 20 and 40 min) were evaluated for the extraction efficiency of the two target compounds. Compared with refluxing extraction, the sonication method was found to be more efficient for both of the components. The sonication with chloroform exhibited similar extraction efficiency to extraction with methanol, but more impurities were observed in methanol extract. Consequently, the samples were extracted with chloroform by sonication for 10,20 , and $40 \mathrm{~min}$ to screen optimal extraction time. The results showed that two markers compounds could be entirely extracted within 20 and $40 \mathrm{~min}$ [8]. Therefore, $20 \mathrm{~min}$ was selected as an appropriate extraction time.

\section{Method Validation}

\section{Specificity}

Specificity of the method was evaluated through the analysis of the blank sample. Possible interference present in the blank sample solution was analyzed by the described HPLC method and compared with the chromatograms of costunolide and dehydrocostuslactone reference sample (Fig. 2). No interfering peaks could be detected at the retention time of costunolide and dehydrocostuslactone.

\section{Linearity, Limits of Detection and Limits of Quantification}

Linear calibration plots were obtained over seven concentration levels. The results showed a good linear correlation $\left(R^{2}>0.9999\right)$ between the peak area and concentration (Table 2 ) over the investigated concentration ranges $(7-280 \mu \mathrm{g} / \mathrm{mL})$.
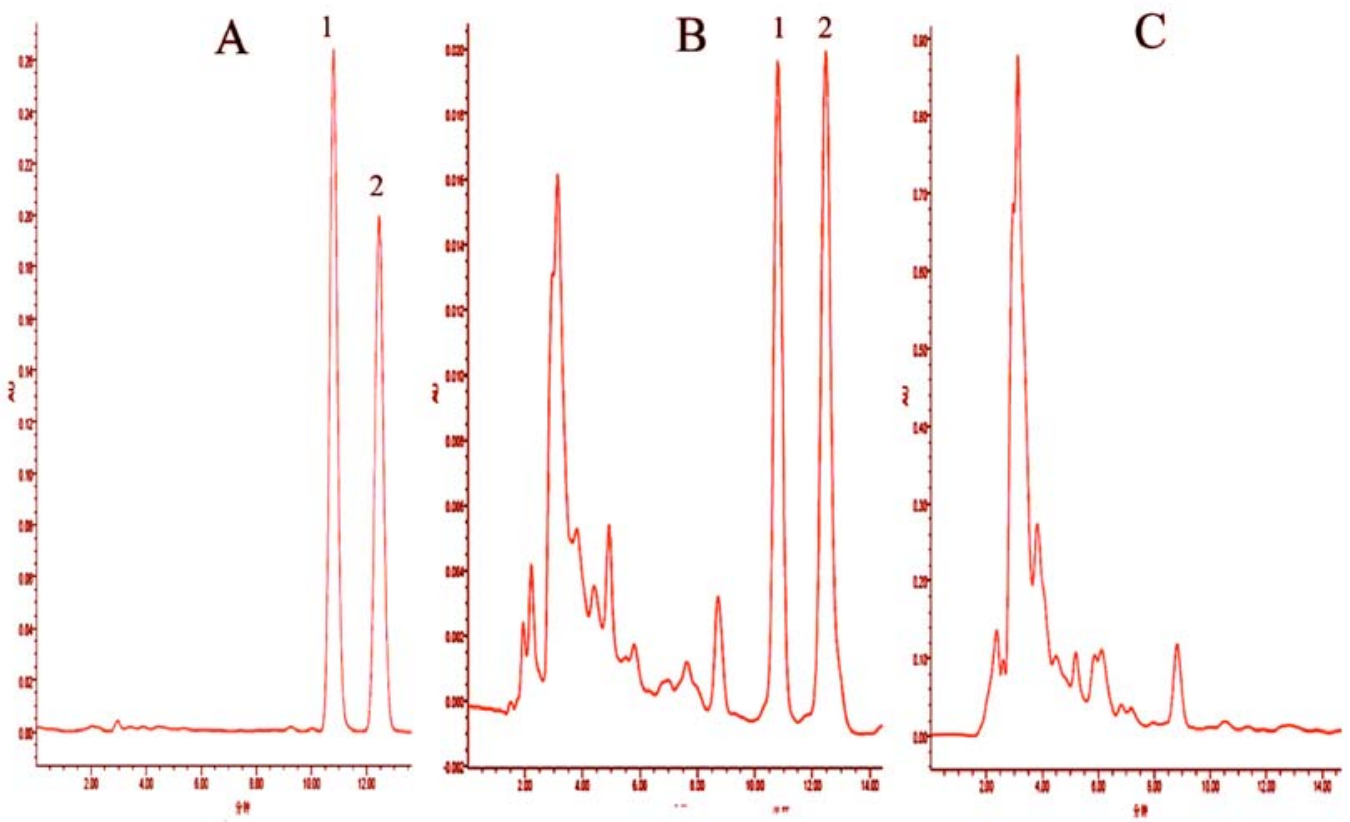

Fig. (2). HPLC of reference substances (A), Xin-ke-shu sample (B), sample of Xin-ke-shu without Radix Aucklandiae (C), 1. costunolide, 2. dehydrocostus lactone. 
Table 2. Regression Equations, Linearity Range, LODs, LOQs, Precision, Repeatability, and Stability Tests (RSD \%) for 2 Analytes Analyzed with the Established HPLC Method

\begin{tabular}{|c|c|c|c|c|c|c|c|c|c|}
\hline \multirow{2}{*}{ Analytes } & \multirow{2}{*}{ Regression Equation } & \multirow{2}{*}{$\begin{array}{c}\text { Correlation } \\
\text { Coefficient } \\
\left(\mathbf{r}^{2}\right)\end{array}$} & \multirow{2}{*}{$\begin{array}{c}\text { Linearity } \\
\text { Range } \\
(\mu \mathrm{g})\end{array}$} & \multirow{2}{*}{$\begin{array}{r}\text { LOD } \\
(\mu \mathrm{g})\end{array}$} & \multirow{2}{*}{$\begin{array}{r}\text { LOQ } \\
(\mu \mathrm{g})\end{array}$} & \multicolumn{2}{|c|}{ Precision } & \multirow{2}{*}{$\begin{array}{c}\text { Repeatability } \\
\quad(n=6)\end{array}$} & \multirow{2}{*}{$\begin{array}{c}\text { Stability } \\
(n=6)\end{array}$} \\
\hline & & & & & & $\begin{array}{l}\text { Intra-day } \\
\quad(n=6)\end{array}$ & $\begin{array}{c}\text { Inter-day } \\
(\mathbf{n}=\mathbf{3})\end{array}$ & & \\
\hline Costunolide & $\begin{array}{c}\mathrm{Y}=1.9044 \times 10^{6} \mathrm{X}+ \\
1781.7\end{array}$ & 1 & $0.07-2.8$ & 0.007 & 0.07 & $0.5 \%$ & $2.68 \%$ & $2.32 \%$ & 2.32 \\
\hline $\begin{array}{l}\text { Dehydrocostu } \\
\text { slactone }\end{array}$ & $\begin{array}{c}\mathrm{Y}=1.5593 \times 10^{6} \mathrm{X}+ \\
2006.7\end{array}$ & 1 & $0.07-2.8$ & 0.014 & 0.07 & $0.65 \%$ & $2.16 \%$ & $2.45 \%$ & 2.13 \\
\hline
\end{tabular}

Note: in the regreesion equation, $\mathrm{X}$ is the peak area, $\mathrm{Y}$ is the concentration of each analyte $(\mu \mathrm{g})$, and $\mathrm{r}$ is the correlation coefficient, LOD is the limit of detection, LOQ is the limit of quantification.

Table 3. The Recovery of Determination of Costunolide and Dehydrocostuslactone

\begin{tabular}{|c|c|c|c|c|c|}
\hline \multirow{2}{*}{ Analyte } & Initial Amount/mg & Added Amount/mg & $\begin{array}{c}\text { Detected } \\
\text { Amount/mg }\end{array}$ & Recovery/\% \\
\hline \hline & & 0.437 & 0.985 & 100.4 & 0.78 \\
\hline Costunolide & 0.544 & 0.541 & 1.075 & 1.59 \\
\hline & & 0.649 & 1.202 & 100.8 & 0.49 \\
\hline Dehydrocostuslactone & 0.778 & 0.615 & 1.394 & 100.1 & 99.7 \\
\hline
\end{tabular}

The stock solutions were diluted to a series of working reference solutions with methanol, and an aliquot of the diluted solutions were injected into HPLC for analysis. LOD and LOQ under the present chromatographic conditions were determined at a signal-to-noise ratio $(\mathrm{S} / \mathrm{N})$ of around 3 and 10, respectively. As shown in Table 2, LOD and LOQ of the analytes were no more than $0.014 \mu \mathrm{g}$ and $0.07 \mu \mathrm{g}$, respectively.

\section{Precision, Reproducibility and Stability}

The precision study used the same sample (batch number: 100537) and followed the validated procedure for sample preparation. The precision of the assay method was evaluated by carrying out six independent assays on three consecutive days. The \% RSD of two analytes was determined to be within the acceptable limit of $5.0 \%$. Multiple injections illustrated that the results were highly reproducible and had a low standard deviation. The RSD of assay results obtained in inter-day and intra-day study (Table 2) was within $3 \%$ and showed a maximum of $2.68 \%$ for costunolide in inter-day, and $0.65 \%$ for dehydrocostuslactone in intra-day studies, confirming a good precision of the developed method.

\section{Recovery}

The accuracy of the method was determined by adding different concentrations of costunolide and dehydrocostuslactone into the sample with a known amount of two standard compounds. The percentage recovery ranged from $99.1 \%$ to $100.8 \%$. The RSD values of each concentration level were not more than $1.56 \%$ (Table 3 ).

\section{Determination of Sample Content}

The validated HPLC method was subsequently applied to simultaneous determination of costunolide and dehydrocostuslactone in 13 samples of Xin-ke-shu preparations. The concentrations of the two markers were calculated by external standard method. The results (Table 4) showed that the total concentrations of two markers were among $0.088-1.317 \mathrm{mg} / \mathrm{g}$. It may due to the difference of the content of Radix Aucklandiae in the preparations and operation of production process. The content of dehydrocostuslactone was always higher than that of costunolide in all samples even eight times higher in sample Sh5. However, in samples Sh1, Ch1, Sh4, H2, and J, there were remarkable differences among the contents of the chemical markers analyzed in different samples, even the contents of costunolide and dehydrocostuslactone varied significantly in different batches from the same pharmaceutical manufacturer. For example, costunolide was abundant in the batches of Sh2 and Sh3, but lower in the batches of Sh1, Sh4, and Sh5. Moreover, the content of costunolide in batch Sh2 is three times higher than that in batch Sh1, both of which produced from the same company. This variation might be due to the quality of Radix Aucklandiae. Contents of constituents from the roots of Radix Aucklandiae will be different because of the weather and geographical location, and even production process will result in the loss of the constitutents in Radix Aucklandiae.

\section{CONCLUSIONS}

An analytical reversed-phase HPLC method was developed and validated thoroughly for simultaneous 
Table 4. The Contents of Costunolide and Dehydrocostuslactone in Xin-ke-shu Preparations (n=3)

\begin{tabular}{|c|c|c|c|c|c|}
\hline Sample No. & Costunolide (mg/g) & RSD $(\%)$ & Dehydrocostuslactone (mg/g) & $\operatorname{RSD}(\%)$ & $\begin{array}{c}\text { Total of Two } \\
\text { Analytes (mg/g) }\end{array}$ \\
\hline Sh1 & 0.180 & 2.17 & 0.355 & 2.97 & 0.535 \\
\hline $\mathrm{Sh} 2$ & 0.542 & 2.32 & 0.775 & 2.45 & 1.317 \\
\hline Sh3 & 0.449 & 1.63 & 0.664 & 2.65 & 1.113 \\
\hline$S$ & 0.458 & 0.95 & 0.674 & 0.91 & 1.132 \\
\hline Ch1 & 0.130 & 2.03 & 0.259 & 1.68 & 0.389 \\
\hline $\mathrm{Ch} 2$ & 0.202 & 2.16 & 0.378 & 1.61 & 0.580 \\
\hline Sh4 & 0.143 & 2.42 & 1.100 & 2.23 & 1.243 \\
\hline Sh5 & 0.096 & 1.81 & 0.819 & 0.56 & 0.915 \\
\hline H1 & 0.451 & 0.22 & 0.584 & 0.91 & 1.035 \\
\hline $\mathrm{H} 2$ & 0.030 & 3.13 & 0.058 & 4.56 & 0.088 \\
\hline $\mathrm{Y}$ & 0.206 & 2.22 & 0.328 & 2.61 & 0.534 \\
\hline$X$ & 0.238 & 2.91 & 0.401 & 0.89 & 0.639 \\
\hline $\mathrm{J}$ & 0.045 & 3.85 & 0.124 & 2.79 & 0.169 \\
\hline
\end{tabular}

determination of costunolide and dehydrocostuslactone in Xin-ke-shu preparations. The developed method is sensitive, simple and specific and can be employed for monitoring the quality of the roots of Radix Aucklandiae (Mu-Xiang). This established method, combined with our previous established procedure [5], provided a comprehensive strategy for quality control of Xin-ke-shu preparations.

\section{ACKNOWLEDGEMENTS}

This work was financially supported by the National Natural Science Foundation of China (No. 81073021) and Beijing Natural Science Foundation (No. 7112093).

\section{CONFLICT OF INTEREST}

None declared

\section{ABBREVIATIONS}

$\begin{array}{lll}\text { HPLC } & =\quad \begin{array}{l}\text { High Performance Liquid } \\ \text { Chromatography }\end{array} \\ \text { LC-LTQ-Orbitrap = } & \begin{array}{l}\text { Liquid Chromatography Coupled } \\ \text { with Electrospray Ionization } \\ \text { Hybrid Linear Trap Quadrupole } \\ \text { orbitrap }\end{array}\end{array}$

LOD

LOQ

TCM

$\begin{array}{ll}= & \text { Limit of Detection } \\ = & \text { Limit of Quantification } \\ = & \text { Traditional Chinese Medicine }\end{array}$

\section{REFERENCES}

[1] Lin, S.Y.; Zhang, G.F. The research of XinKeShu tablet. Chin. J. Integr. Med. Cardio-/cerebrovasc. Dis., 2009, 7 (37), 818-820.

[2] X.F. Li.A. RP-HPLC determination of content of puerarin in Xinkeshu tablet. J. Zhejiang. Univer. Tradi. Chin. Med., 2008, 32 (4), 534-535.

[3] Zhang, D.M.; Liu, B.; Sang, Y. HPLC determination of content of puerarin and tanshinone IIA. Chin Pharm., 2003, 23 (5), 41-42.

[4] Jia, S.X.; Liu, L.C.; Wu, J.M. HPLC determination of content of protocatechuic aldehyde. Chin. J. Hosp. Pharm 2003, 23(5), 286287.

[5] Peng, J.B.; Jia, H.M.; Liu, Y.T. Qualitative and quantitative characterization of chemical constituents in Xin-Ke-Shu preparations by liquid chromatography coupled with a LTQ Orbitrap mass spectrometer. J. Pham. Biomed. Anal., 2011, 55, 984-995.

[6] Li, Z.Y.; Pan, Y.; Ding, G. Qualitative and quantitative studies of costunolide and dehydrocostus lactone in Radix Aucklandiae. Lish. Med. Mat. Med. Res., 2004, 15 (11), 745.

[7] The State Pharmacopoeia Commission of the People's Republic of China. Pharmacopoeia of the People's Republic of China. Chemical Industry Press: Beijing, 2005.

[8] Liu, J.H.; Li, L.H.; Wu, X.X. A preliminary study of factors affecting costunolide and dehydrocostus lactone in Radix Aucklandiae. Lish. Med. Mat. Med. Res., 2009, 20(12), 3013-3014. 